\title{
Modelling of dust around the symbiotic Mira RR Telescopii during obscuration epochs
}

\author{
T. Jurkic and D. Kotnik-Karuza
}

\author{
Department of Physics, University of Rijeka, Omladinska 14, 51000 Rijeka, Croatia \\ e-mail: tjurkic@phy.uniri.hr
}

Received 4 January 2012 / Accepted 29 March 2012

\begin{abstract}
Context. Symbiotic Miras represent a class of peculiar binaries whose nature is still not well understood. Physical properties of the circumstellar dust and associated physical mechanisms play an important role in understanding the evolution of symbiotic binaries and the interaction between their components. We present a model of inner dust regions around the cool Mira component of the symbiotic nova RR Tel based on the near-IR terrestrial photometry and infrared ISO spectra.

Aims. Our goal is to find a comprehensive and consistent model of the circumstellar inner dust regions around the Mira component that can explain the observed photometric and spectroscopic features in the near- and mid-infrared.

Methods. Available JHKL photometric observations from South African Astronomical Observatory were collected and corrected for Mira pulsations as well as for interstellar reddening to follow temporal changes of the near-infrared colours. Spectral energy distributions (SEDs) from 1 to $13 \mu \mathrm{m}$ during obscuration epoch were reconstructed with the simultaneously available ISO/SWS spectra and JHKL magnitudes. The dust properties were determined by modelling both the reconstructed SEDs and the near-IR colours using the DUSTY numerical code. This $1 \mathrm{D}$ code solves radiative transfer through the circumstellar dust by calculating the dust temperature profile assuming spherical symmetry.

Results. The Mira pulsation period of 387 days was found and confirmed with two independent fitting methods. A long-term variation of 7000 days, which cannot be attributed to orbital motion, was obtained from the analysis of the near-IR magnitudes. Reconstructed infrared SEDs were modelled successfully by a single dust shell with dust distribution enhanced by radiatively driven stellar winds. Mira temperature, dust sublimation temperature, grain diameter, density distribution, and optical depth have been obtained. Our model shows a maximum dust grain diameter of $4 \mu \mathrm{m}$, which is larger than expected and can be explained by grain growth in conditions of increased mass loss during obscuration epochs. Obscurations in the near-IR can be understood as a result of change of the dust optical depth and of the mass loss rate of Mira component. Change of the dust temperature on the inner boundary of the dust shell with pulsation phase have been identified by SED modelling. Assuming a gas-to-dust ratio of 200, we found a variable mass loss rate between 2.3 and $9.0 \times 10^{-6} M_{\odot} / \mathrm{yr}$ and estimated the distance to RR Tel to be $2.7 \mathrm{kpc}$. Our results suggest a relatively low influence of the hot component on circumstellar dust and strong shielding of dust from the UV flux.
\end{abstract}

Key words. binaries: symbiotic - circumstellar matter - radiative transfer - stars: AGB and post-AGB - infrared: stars

\section{Introduction}

Symbiotic stars are binary stellar systems in the late stage of stellar evolution that consist of a cool red giant or a Mira star and a hot compact companion with large mass transfer from the giant star toward the companion (see Kenyon 1986). Around $80 \%$ of the symbiotic stars contain a normal giant and are classified as S-type symbiotics. The remainder contain Mira variables in which near-IR observations show a reddened Mira component surrounded by circumstellar dust at a temperature of about $1000 \mathrm{~K}$. These symbiotics with warm dust shells are classified as D-type symbiotics (Mikolajewska et al. 1988; Kenyon 1986). The warm thick dust shell is usually responsible for the nearinfrared excess and obscuration events, most likely caused by a change in optical depth of the dust envelope (Kotnik-Karuza et al. 2006; Whitelock 2003).

$\mathrm{RR}$ Tel is a D-type symbiotic nova that underwent its last nova-like outburst in 1944. It is a detached interacting binary system of relatively wide spatial separation, consisting of a cool Mira component and a hot compact star, probably a white dwarf (Whitelock 2003). The studies of this binary system started about 60 years ago (Mayall 1949; Thackeray 1950). Some general properties of RR Tel are given in Table 1.
Table 1. General properties of RR Tel.

\begin{tabular}{lcc}
\hline \hline Parameter & Value & References \\
\hline Spectral class & Mira M6/M5 & 1,2 \\
$A_{V}$ & 0.3 & 3,4 \\
$V$ (mag) & 10.8 & 5 \\
$d$ (kpc) & 2.5 & 6 \\
$P$ (days) & 385 & 7 \\
& 387 & 8 \\
\hline
\end{tabular}

References. (1) Muerset \& Schmid (1999); (2) Allen (1980); (3) Penston et al. (1983); (4) Young et al. (2005); (5) Belczynski et al. (2000); (6) Kotnik-Karuza et al. (2006); (7) Gromadzki et al. (2009); (8) Feast et al. (1983).

However, full explanations of all spectroscopic and photometric features, as well as a satisfactory model of RR Tel that includes all relevant physical mechanisms are still lacking. For a complete dynamical model of symbiotic Miras, multiwavelength and long-term observations are needed. Up to now, most modelling attempts were focused mainly on the line-emitting nebular region dominated by heating from the hot component with 
inclusion of shock-waves (Contini \& Formiggini 1999) or accretion disks (Lee \& Park 1999).

Attempts to fully explain the near-infrared spectra and to describe physical properties of the circumstellar dust shell of the Mira component are rare and mostly based on the blackbody model of a thick dust shell of constant temperature, without determination of any physical dust properties such as grain size or sublimation temperature. Use of radiative transfer to model the dust properties was merely confined to single objects (e.g. Tuthill et al. 2000; Bryan \& Kwok 1991). The interest in such studies has recently been revived as a result of advances in instruments and techniques (e.g. Karovicova et al. 2011; Sacuto $\&$ Chesneau 2009). More recently, Angeloni et al. (2010) reconstructed the spectral energy distribution (SED) from the visual to the far-infrared and modelled it with their own code SHUMA. The code includes both shock-waves induced by collision of stellar winds of the two components and heating of the thin layer in the circumstellar dust shell caused by the hot component. According to their model, only the thin dust shell region close to the shock front is heated and emits a strong infrared flux as a blackbody. Their model includes two circumstellar dust shells, with the inner shell surrounding only the cool Mira star and the outer, circumbinary shell at a temperature of $400 \mathrm{~K}$ and with an emission peak intensity at about $10 \mu \mathrm{m}$. Because the shape of the spectra and wavelength of the peak emission correspond to the silicon feature found in many circumstellar dust shells, there is a shadow of doubt about the validity of this two-shell model.

In this work we are going to determine the circumstellar dust properties of the Mira component with the DUSTY code, which takes into account the advantages of the scale invariance of radiative transfer. The Mira temperature will be obtained independently, using the near-IR colour indices to reduce the number of free fitting parameters. The dust properties will be determined by modelling the SED between 1 and $13 \mu \mathrm{m}$ reconstructed from the near-IR terrestrial observations and the ISO/SWS spectra. The analysis of long-term variations in the near-IR requires reducing the light curves for Mira pulsations, which in turn makes it necessary to determine the pulsation period. This procedure will enable us to look for possible differences in the pulsation parameters between epochs with and without obscuration. The time evolution of dust parameters will be established from the corresponding behaviour of the near-IR colours. We will estimate the mass loss and distance from the obtained dust properties.

\section{Observations and methods}

To investigate the dust properties of RR Tel, we used two sets of data: long-term near-infrared JHKL photometric magnitudes (Kotnik-Karuza et al. 2006; Gromadzki et al. 2009) and publicly available near- to mid-infrared ISO/SWS spectra (Sloan et al. 2003). The near-infrared JHKL magnitudes of RR Tel observed from 1975 to 2003 from the South African Astronomical Observatory (SAAO) have been analyzed in view of the long-term light curve variations (Kotnik-Karuza et al. 2006). Photometric magnitudes were obtained prior to 1984 on the Glass system (Glass 1973) and after 1984 on the Carter system (Carter 1990). However, all observations made on the Glass system were later put on the Carter system.

\subsection{Correction of light curves by reducing Mira pulsations and determining the pulsation period}

To determine the circumstellar dust properties, the light curves were corrected for interstellar reddening and Mira pulsations were reduced with our own code. The Mira pulsation period was determined with the phase dispersion minimization (PDM) technique as described in Lafler \& Kinman (1965), which allows determining any periodicity regardless of the light-curve shape. In this method, only the existence of periodicity is important. The pulsation period is the only parameter to be determined, which reduces the number of free parameters usually fitted with other techniques. The method consists of subdividing the observational times in bins of different length, from which the phase diagram is determined. The length of the bins and the pulsation period were fitted until least scattering in the phase diagram was achieved. The phase diagram was then fitted to the third-order Fourier polynomial and subtracted from observations, resulting in the light curve corrected for Mira pulsations. The pulsation amplitude and starting epoch for the light curve variation can also be determined for the best-fitted phase diagram.

We also fitted a sinusoidal function using the standard $\chi^{2}$ method to determine the dominant influence in light-curve variations. To additionally constrain the number of fitted parameters, first we determined the exact time of each clearly observed maximum and minimum magnitude in the light curve using the best fit to the third-order Fourier polynomial. Only those parts of the light curve with observations at both sides of the clearly present extremes (minimum or maximum) in the light curve were used for determining the times of maxima and minima. These times were shifted according to the fitted period to the time before the first observation, yielding the starting epoch for the light curve variation. After fixing the starting epoch, the period and amplitudes of the pulsations were fitted by minimizing the $\chi^{2}$.

The corrected JHKL magnitudes show only long-term variations and give clear evidence of obscuration events (Kotnik-Karuza et al. 2006).

\subsection{Reconstruction of the spectral energy distribution (SED)}

The spectral energy distribution of the symbiotic Mira RR Tel was reconstructed at different time intervals, when both $J H K L$ magnitudes and ISO short wavelength spectra were available simultaneously.

The exact reconstruction of SEDs that include JHKL photometric magnitudes proved to be quite a challenge. JHKL magnitudes were not available at the time when ISO spectra were obtained, therefore we had to interpolate $J H K L$ values at the time when ISO spectra were observed. Interpolation was achieved through the fit of nearby $J H K L$ magnitudes on thirdorder Fourier polynomial at about the time of ISO/SWS observations (Table 2). The ISO/SWS 54601206 spectrum (Fig. 5a) was taken only six days before the near-IR photometric observations and consequently the reconstructed SED is much more accurate and reliable than the 73402079 spectrum. Thus, JHKL errors in the reconstructed SED 54601206 are close to the instrumental error of $0.02 \mathrm{mag}$. Although ISO spectra in some cases have a problem with absolute calibration, the photometric $L$ magnitude of 3.31 is surprisingly almost the same as the $L$ magnitude of 3.34 calculated from the ISO/SWS spectrum. In contrast, the ISO/SWS spectrum 73402079 (Fig. 5b) was taken 100 days apart from the nearest $J H K L$ observation, so the near-IR part of the reconstructed SED is less reliable, with $J H K L$ errors of the order of $0.15 \mathrm{mag}$. Also, determining the JHKL magnitudes at the epoch when this ISO spectrum was taken was not possible through a straightforward fit of the JHKL light curves, because small changes in the fit parameters yielded significant variation in the fitted JHKL magnitudes. Instead, we fitted differences of 
Table 2. Observational data for the spectral energy distribution reconstructed from the ISO/SWS spectra and JHKL SAAO photometry.

\begin{tabular}{lcc}
\hline \hline ISO/SWS number & 54601206 & 73402079 \\
Date & $1997 / 05 / 15$ & $1997 / 11 / 19$ \\
JD & 2450584 & 2450772 \\
$J$ & $7.96 \pm 0.03$ & $7.06 \pm 0.20$ \\
$H$ & $6.36 \pm 0.05$ & $5.56 \pm 0.17$ \\
$K$ & $4.98 \pm 0.05$ & $4.26 \pm 0.15$ \\
$L$ & $3.31 \pm 0.04$ & $2.62 \pm 0.03$ \\
\hline
\end{tabular}

Notes. The near-IR magnitudes for a particular date of ISO/SWS observation were derived using the fit as described in text.

the $J, H$ and $K$ with respect to the $L$ magnitude and used the $L$ magnitude calculated from the ISO/SWS spectrum.

Effects of the filter transmission also have to be taken into account because there is no standard JHKL photometric system. Unfortunately, filter transmission curves for the Carter system were not available, therefore we used transmission curves for the Glass system and for the Bessel \& Brett (1988) universal JHKL photometric system mainly based on Glass (1973) and Carter (1990). Transmission curves for the Glass system are essentially the same as for the Carter system, i.e. the instrument and filters are the same in both systems and differ only in the detector. Indeed, our results show no significant differences if the Bessel \& Brett (1988) or Glass (1973) JHKL filter transmission curves are used. First, integrated $J H K L$ Vega fluxes with given filters were obtained from the SED of Vega (Bohlin 2007). The integrated flux of RR Tel in $L$ band was found from the ISO/SWS SED and compared to the $L$ band flux of the SED of Vega, followed by computation of RR Tel JHKL integrated fluxes. While reconstructing the SED, we also included the influence of the interstellar extinction on the ISO/SWS and on the computed $J H K L$ fluxes. Appendix A describes the mathematical procedure of the SED reconstruction. Statistical weight was also used in suppressing the distortion of the fitted results against the part of the spectra that have many data points in a narrow wavelength region compared to the wavelength region with only few data points. While ISO/SWS observations are mostly equally distributed in wavelengths, the near-infrared part of the SED has only three points obtained from SAAO photometry at $J, H$ and $K$ passbands. To compensate for this distortion, we used the statistical weight $w=1$ for ISO/SWS observations, $w_{J}=380$, $w_{H}=565, w_{K}=720$ and $w_{L}=475$ for $J H K L$ photometric observations calculated as described in Appendix B. It must be emphasized that precise $J H K L$ flux determination from photometric magnitudes is of greatest importance because this part of the spectra is missing from the ISO/SWS SEDs but is crucial in determining the dust properties.

The two reconstructed SEDs (Fig. 5) taken during the observed obscuration epoch span over the near- and mid-infrared spectral region up to $13 \mu \mathrm{m}$. This spectral region contains the dust emission and scattering from the sublimation region around the Mira component up to the colder regions a few sublimation radii away from the star.

\subsection{Theoretical modelling}

The numerical code DUSTY (Ivezic et al. 1999) was used to model the dust properties of the circumstellar dust shell around the cool Mira component. DUSTY solves the radiative transfer problem for dust absorption, emission, and scattering by calculating the dust temperature profile assuming spherical symmetry.
This 1D code takes advantage of the scaling invariance and selfsimilarity theory to constrain the number of free input parameters (Ivezic \& Elitzur 1997). Scaling invariance implies only the scaled distance $y$ with respect to the dust shell inner radius (sublimation radius) $r_{\text {in }}$ as a relevant parameter:

$y=\frac{r}{r_{\text {in }}}$.

Only the shape of the dust density distribution and the spectral shape of the input heating radiation is needed; absolute stellar luminosity does not enter the equations. Starting from the input parameters, the code models the output radiation reprocessed by dust and yields the physical and geometrical properties of the dust shell. Although the code requires spherical symmetry, Vinkovic et al. (2003, 2006) have also shown that it can handle other axially symmetric dust distributions such as flattened haloes.

DUSTY can handle various analytical forms for the dust density distribution, including full dynamical calculation for radiatively driven winds. Various dust chemistries (silicates, carbon, olivine, forsterite, etc.) and the corresponding optical properties are supported or can be entered in the user-supplied files. The dust grain size distribution, the minimum and maximum grain diameters, the dust sublimation temperature, the shell size and the optical depth can be varied to produce an acceptable model. The variation in power index $p$ of the dust density distribution $\eta$

$\eta \propto \frac{1}{y^{p}}$

can give an insight into the dust geometry (spherical shell, flattened halo, disk-like structure).

The circumstellar model with the Mira component in the centre of the dust shell was used, while the input stellar radiation was approximated by a blackbody Mira star at a temperature determined from the $J, H$ and $K$ photometry in an epoch of negligible obscuration. Because Miras are well known to have strong stellar winds that drive the expansion of their envelopes, the analytical approximation for dust density distribution $\eta$ enhanced by radiatively driven winds (Ivezic et al. 1999; Elitzur \& Ivezic 2001)

$\eta \propto \frac{1}{y^{2}} \sqrt{\frac{y}{y-1+\left(v_{\mathrm{i}} / v_{\mathrm{e}}\right)^{2}}}$

was used, where $v_{\mathrm{i}}$ and $v_{\mathrm{e}}$ are initial and terminal wind velocities, respectively. The ratio of initial to final velocity, $\epsilon_{\mathrm{v}}=v_{\mathrm{i}} / v_{\mathrm{e}}$ is practically irrelevant if $\epsilon_{\mathrm{v}} \leq 0.2$ (Ivezic et al. 1999) and DUSTY uses the default value $\epsilon_{\mathrm{v}}=0.2$. This assumption is justified because the initial velocity for our expected sublimation temperatures of $1000-1500 \mathrm{~K}$ is equal to $v_{\mathrm{i}}=2-3 \mathrm{~km} \mathrm{~s}^{-1}$, which follows from Eq. (9) in Elitzur \& Ivezic (2001). Note that to keep the condition $\epsilon_{\mathrm{v}} \leq 0.2$ valid, the minimum value of the terminal velocity should be $15 \mathrm{~km} \mathrm{~s}^{-1}$. We used the value of 200 for gas-to-dust ratio in deriving the mass loss rates and terminal wind velocities. The typical MRN dust grain size distribution (Mathis et al. 1977) $n(a) \propto a^{-q}\left(a_{\min } \leq a \leq a_{\max }\right)$ was applied in the model, with the power index $q=3.5$, the minimum grain size $a_{\min }$ fixed at $0.005 \mu \mathrm{m}$ and the maximum grain size $a_{\max }$ determined by the model. The dust composition typical for Miras containing $100 \%$ warm silicates was assumed (Ossenkopf et al. 1992). The outer dust shell radius was fixed at $20 r_{\text {in }}$, while the dust shell inner radius $r_{\text {in }}$ was obtained by fitting, together with the dust sublimation temperature $T_{\text {dust }}$. 
Table 3. Pulsation parameters determined by fitting sinusoidal function on $J, H, K$ and $L$ light curves in epochs before and after JD 2446352 , i.e. out of and during obscuration events.

\begin{tabular}{lccc|ccc}
\hline \hline & \multicolumn{3}{c|}{ Out of obscuration events } & \multicolumn{3}{c}{ During obscuration events } \\
\hline & $P$ & $\begin{array}{c}\mathrm{JD}_{0} \\
\text { (days) }\end{array}$ & $\begin{array}{c}A \\
(\mathrm{mag})\end{array}$ & $\begin{array}{c}P \\
\text { (days) }\end{array}$ & $\begin{array}{c}\mathrm{JD}_{0} \\
(\text { days })\end{array}$ & $\begin{array}{c}A \\
(\mathrm{mag})\end{array}$ \\
\hline$J$ & $384.3_{-9.3}^{+9.2}$ & $2442240 \pm 20$ & $0.63 \pm 0.28$ & $385.5_{-4.0}^{+4.0}$ & $2442274 \pm 28$ & $0.68 \pm 0.25$ \\
$H$ & $384.2_{-9.2}^{+9.9}$ & $2442248 \pm 15$ & $0.59 \pm 0.27$ & $385.6_{-4.1}^{+4.2}$ & $2442273 \pm 27$ & $0.67 \pm 0.25$ \\
$K$ & $384.5_{-9.5}^{+12.5}$ & $2442243 \pm 14$ & $0.48 \pm 0.28$ & $386.0_{-4.7}^{+4.9}$ & $2442258 \pm 28$ & $0.59 \pm 0.26$ \\
$L$ & $384.4_{-9.4}^{+15.6}$ & $2442240 \pm 11$ & $0.37 \pm 0.28$ & $386.6_{-6.4}^{+7.0}$ & $2442221 \pm 17$ & $0.45 \pm 0.26$ \\
\hline Average & $384.3_{-4.7}^{+5.5}$ & $2442243 \pm 7$ & & $385.8_{-2.3}^{+2.4}$ & $2442247 \pm 12$ & \\
\hline
\end{tabular}

Notes. Averaged values of determined pulsation parameters are given in the last line $\left(P\right.$ - pulsation period; $\mathrm{JD}_{0}-$ starting epoch of maximum brightness; $A$ - half peak-to-peak amplitude).

Table 4. Pulsation parameters determined by fitting of any periodic function on $J, H, K$ and $L$ magnitudes in epochs before and after JD 2446352 , i.e. out of and during obscuration events.

\begin{tabular}{lcccc|cccc}
\hline \hline \multicolumn{4}{c|}{ Out of obscuration events } & \multicolumn{4}{c}{ During obscuration events } \\
\hline & $\begin{array}{c}P \\
\text { (days) }\end{array}$ & $\begin{array}{c}\mathrm{JD}_{0} \\
\text { (days) }\end{array}$ & $\begin{array}{c}A \\
(\mathrm{mag})\end{array}$ & $N$ & $\begin{array}{c}P \\
(\text { days })\end{array}$ & $\begin{array}{c}\mathrm{JD}_{0} \\
(\text { days })\end{array}$ & $\begin{array}{c}A \\
(\mathrm{mag})\end{array}$ & $N$ \\
\hline$J$ & $385.3_{-9.9}^{+5.3}$ & 2442250 & 0.67 & $9_{-5}^{+15}$ & $386.0_{-2.3}^{+1.4}$ & 2442259 & 0.73 & $5_{-1}^{+3}$ \\
$H$ & $383.3_{-8.9}^{+8.2}$ & 2442241 & 0.58 & $7_{-3}^{+17}$ & $386.0_{-6.7}^{+2.0}$ & 2442244 & 0.70 & $5_{-1}^{+4}$ \\
$K$ & $387.1_{-12.7}^{+9.4}$ & 2442232 & 0.56 & $10_{-7}^{+14}$ & $386.6_{-0.9}^{+2.9}$ & 2442228 & 0.57 & $10_{-5}^{+0}$ \\
$L$ & $386.0_{-19.6}^{+10.5}$ & 2442250 & 0.39 & $23_{-20}^{+11}$ & $386.6_{-0.8}^{+3.8}$ & 2442217 & 0.45 & $10_{-5}^{+0}$ \\
\hline Average & $385.1_{-5.6}^{+3.8}$ & $2442243 \pm 4$ & & & $386.3_{-0.6}^{+1.0}$ & $2442237 \pm 9$ & & \\
\hline
\end{tabular}

Notes. Averaged values of determined pulsation parameters are given in the last line $\left(P\right.$ - pulsation period; $\mathrm{JD}_{0}-$ starting epoch of maximum brightness; $A$ - half peak-to-peak amplitude; $N$ - number of bins).

We fitted all relevant dust properties such as dust sublimation temperature, dust density distribution, optical depth, and maximum grain size. The dust properties were incremented in steps of $100 \mathrm{~K}$ for dust sublimation temperature, of 0.2 for optical depth and of $0.25 \mu \mathrm{m}$ for maximum grain size diameter. In addition to correcting for the radiatively driven winds, the models were also fitted using three different values of power index $p(1,1.5$ and 2$)$. We also corrected all fluxes for interstellar extinction $A_{\mathrm{V}}$ equal to 0.3 (Penston et al. 1983; Young et al. 2005).

\section{Results and discussion}

\subsection{Mira pulsation period in the infrared}

We determined and confirmed the pulsation period of the Mira component with two fitting techniques on near-IR photometric magnitudes: a sinusoidal fit, and a more complex fit of any periodic function, as described in Sect. 2.1 The results of both techniques for the epoch of minimum obscuration before JD 2446352 and for the following epoch after JD 2446352 that is characterized by obscuration events are shown in Tables 3 and 4 , respectively. Table 5 contains pulsation parameters determined over the whole 28 -year observed period regardless of obscuration level.

Determination of pulsation parameters before JD 2446352 (out of obscuration events) and after JD 2446352 (during obscuration events) was carried out to determine possible differences in the behaviour of the stellar atmosphere during these two epochs. Differences between pulsation periods and/or change in pulsation phases or amplitudes, when the Mira star enters an epoch with significant obscuration, can point to a change of the behaviour of the Mira pulsations. This change in pulsations might cause a release of additional dust from the outer atmospheric layers and consequently lead to higher obscuration in the near-infrared. However, regardless of the applied fitting method (Tables 3 and 4), no significant change in pulsation parameters (period, phase and amplitude) has been obtained. Moreover, there are no differences in pulsation parameters for different near-IR photometric bands.

Because the two analysed epochs cannot be distinguished with respect to the pulsation parameters, we applied the two fitting techniques on the whole 28-year observed interval (Table 5). After allowing for error limits, there are no significant differences between the light curve fits with the sinusoidal function and with an arbitrary periodic function. This means that the light curve variations in the infrared are predominantly sinusoidal, which is typical of Mira variables.

Our pulsation periods of 386.9 and 387.1 days obtained for the whole 28-year epoch using the two fitting techniques agree well with the pulsation period of $385 \pm 4$ days (Gromadzki et al. 2009) and of 387 days (Feast et al. 1983) although different approaches and methods were applied.

$J H K L$ colours corrected for Mira pulsations obtained by the two fitting techniques are shown in Fig. 1. The differences between the corresponding colours are plotted as relatively small residua in the lower part of each diagram. Because the first method fits the sinusoidal function and the second the periodic function of arbitrary shape, the residua can show possible nonsinusoidal or long-term variations. There is an indication of a possible long-term pulsation trend in all colours. Sinusoidal fits of residua obtained from the colour indices (Fig. 1) and from 
T. Jurkic and D. Kotnik-Karuza: Modelling of dust around RR Tel during obscuration epochs

Table 5. Pulsation parameters determined over the whole 28-year observed period using the two fitting techniques on the $J, H, K$ and $L$ light curves.

\begin{tabular}{lccc|cccc}
\hline \hline & \multicolumn{3}{c|}{ Sinusoidal function } & \multicolumn{4}{c}{ Periodic function } \\
\hline & $P$ & $\begin{array}{c}\mathrm{JD}_{0} \\
\text { (days) }\end{array}$ & $\begin{array}{c}A \\
(\mathrm{mag})\end{array}$ & $\begin{array}{c}P \\
(\text { days })\end{array}$ & $\begin{array}{c}\mathrm{JD}_{0} \\
(\text { days })\end{array}$ & $\begin{array}{c}A \\
(\mathrm{mag})\end{array}$ & $N$ \\
\hline$J$ & $386.9_{-2.1}^{+2.0}$ & $2442238 \pm 27$ & $0.68 \pm 0.19$ & $387.1_{-1.7}^{+0.9}$ & 2442242 & 0.72 & $8_{-4}^{+9}$ \\
$H$ & $386.8_{-2.2}^{+2.1}$ & $2442244 \pm 24$ & $0.65 \pm 0.18$ & $387.1_{-1.8}^{+1.3}$ & 2442245 & 0.68 & $8_{-4}^{+12}$ \\
$K$ & $386.8_{-2.5}^{+2.4}$ & $2442238 \pm 24$ & $0.56 \pm 0.19$ & $387.2_{-1.7}^{+1.4}$ & 2442235 & 0.59 & $9_{-5}^{+11}$ \\
$L$ & $387.0_{-3.3}^{+3.2}$ & $2442220 \pm 17$ & $0.43 \pm 0.19$ & $386.9_{-3.2}^{+2.3}$ & 2442230 & 0.39 & $10_{-7}^{+10}$ \\
\hline Average & $386.9_{-1.2}^{+1.1}$ & $2442232 \pm 11$ & & $387.1_{-1.0}^{+0.6}$ & $2442238 \pm 3$ & & \\
\hline
\end{tabular}

Notes. Averaged values of determined pulsation parameters are given in the last line $\left(P\right.$ - pulsation period; $\mathrm{JD}_{0}-$ starting epoch of maximum brightness; $A$ - half peak-to-peak amplitude; $N$ - number of bins).
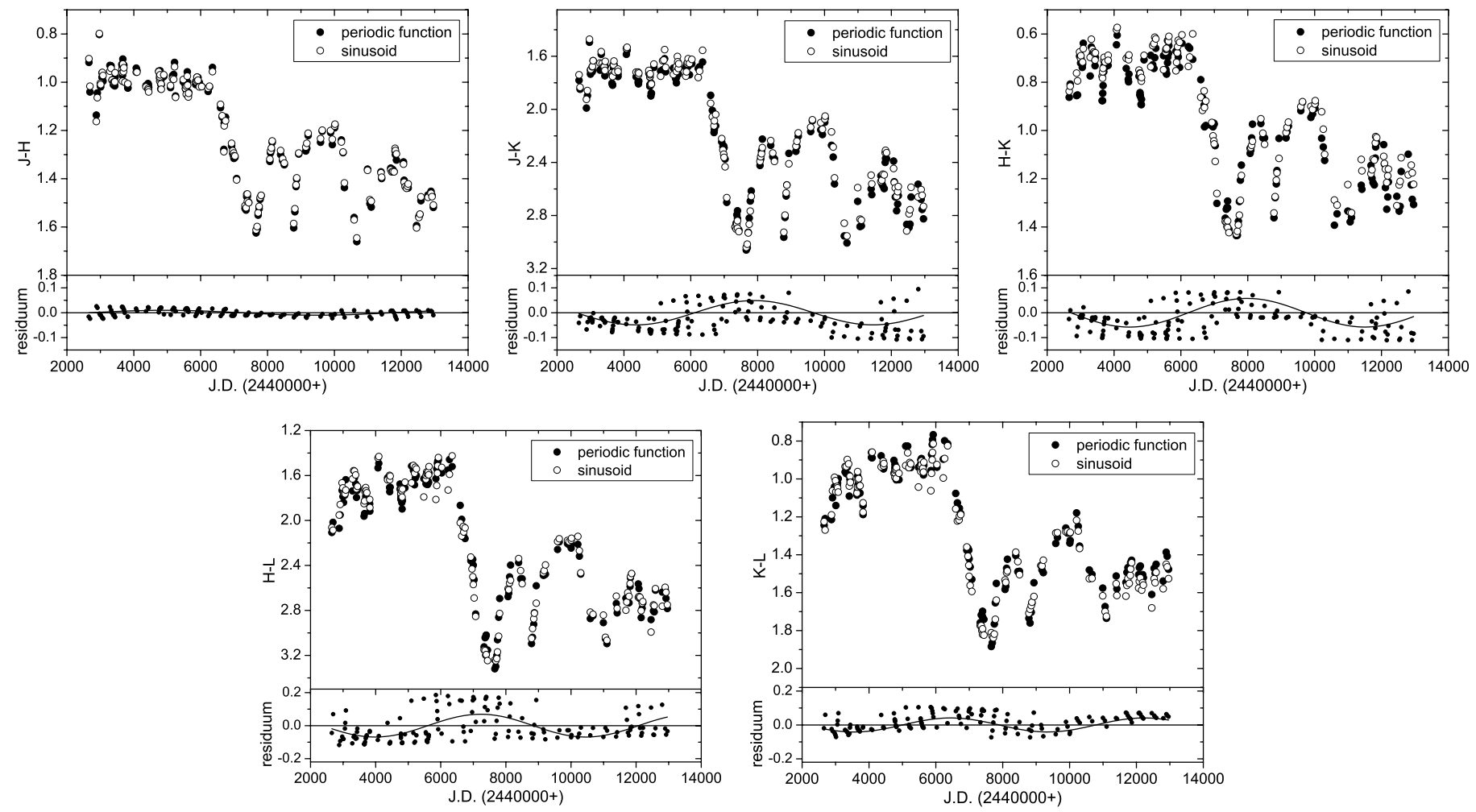

Fig. 1. Colours of RR Tel corrected for Mira pulsations with the two fitting methods. Residua as a difference between the fit with a sinusoidal and with an arbitrary periodic function are shown at the bottom of each diagram. The sinusoidal fit of the residua yields a period of long-term pulsations of about 7100 days.

the JHKL magnitudes (Fig. 2) yield a period of $\sim 7100$ days ( $\sim 20$ years) and $\sim 6450$ days ( $\sim 18$ years) respectively (Table 6 ). Both values agree well within a one sigma error. The long-term pulsation trend may be connected with orbital motion of the two components, assuming a total mass of the system of $2.5 M_{\odot}$ and a distance between the components of about 8-10 AU. However, expected values of at least 25-30 AU for the separation between the components in detached systems lead to orbital periods of around 100 years (Gromadzki et al. 2009). Therefore, an explanation of origin of this long-term variation by orbital motion is highly unlikely and another mechanism should be searched for. Note as well that the long-term variation in the $L$ magnitude seems to be in anti-phase with $J, H$ and $K$ variations (Fig. 2), which may be related to circumstellar dust, because the $L$ band, in contrast to the $J, H$ and $K$ bands, is generally dominated by dust emission.

The JHKL light curves corrected for Mira pulsations are shown in Fig. 3, where three distinct obscuration events are the most outstanding features.

Pulsation ephemerids (times of maximum brightness) for RR Tel can be given as

$\max (J H K L)=2442238( \pm 3)+387.1( \pm 0.8) \cdot E$,

where $E$ denotes the number of full cycles that have passed from the time marked as a starting epoch with maximum $J H K L$ brightness.

Furthermore, because the short-term Mira pulsations are predominantly sinusoidal, they can be removed and the $J H K L$ 


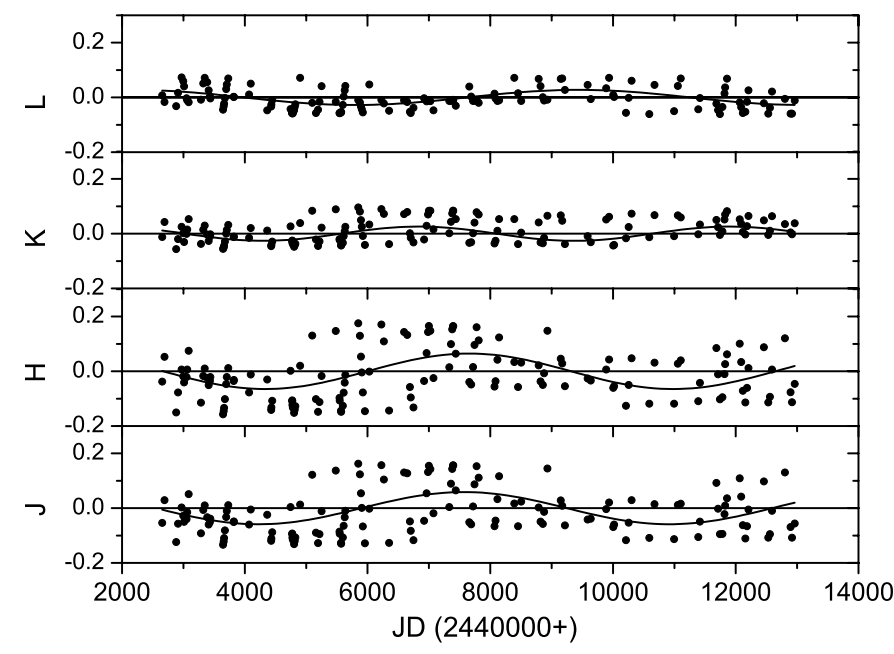

Fig. 2. Differences between $J H K L$ magnitudes corrected for Mira pulsations using fits with the sinusoidal function and with an arbitrary periodic function, yielding a long-term variation of the order of about 6450 days.

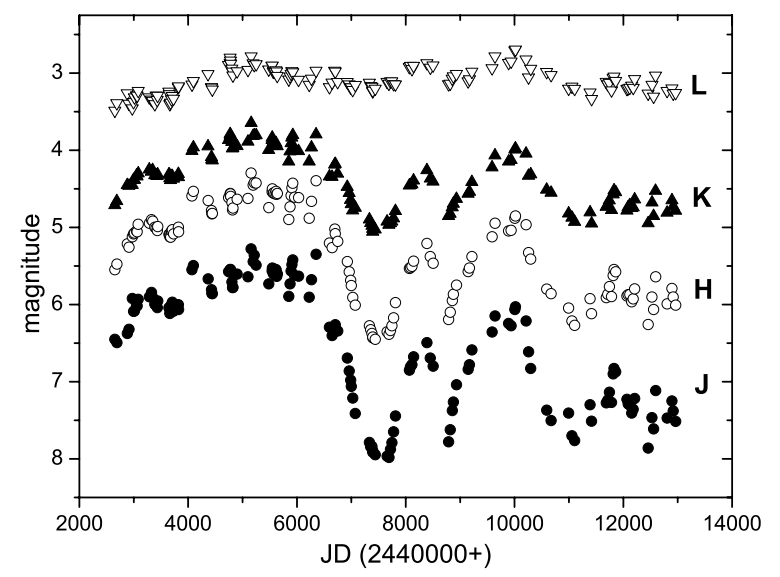

Fig. 3. JHKL light curves obtained from the SAAO observations after removing the Mira pulsations.

Table 6. Long-term pulsation periods determined from the JHKL magnitudes and colour indices.

\begin{tabular}{lc}
\hline \hline Method & $\begin{array}{c}\text { Period } \\
\text { (days) }\end{array}$ \\
\hline $\begin{array}{l}\text { colour indices } \\
\text { JHKL mag. }\end{array}$ & $64090 \pm 540$ \\
\end{tabular}

photometric magnitudes can be corrected as

$$
\begin{aligned}
J^{\prime} & =J-0.68 \cdot \sin (2 \pi \cdot(t-2442335) / 386.9) \\
H^{\prime} & =H-0.65 \cdot \sin (2 \pi \cdot(t-2442340) / 386.8) \\
K^{\prime} & =K-0.56 \cdot \sin (2 \pi \cdot(t-2442335) / 386.8) \\
L^{\prime} & =L-0.43 \cdot \sin (2 \pi \cdot(t-2442317) / 387.0),
\end{aligned}
$$

where $J^{\prime}, H^{\prime}, K^{\prime}$ and $L^{\prime}$ stand for the corrected $J H K L$ magnitudes, $J, H, K$ and $L$ are uncorrected magnitudes and $t$ is time of observation expressed in Julian days.

\subsection{Temperature determination of the Mira component}

Independent determination of the temperature of the Mira component plays an important role in modelling the dust properties

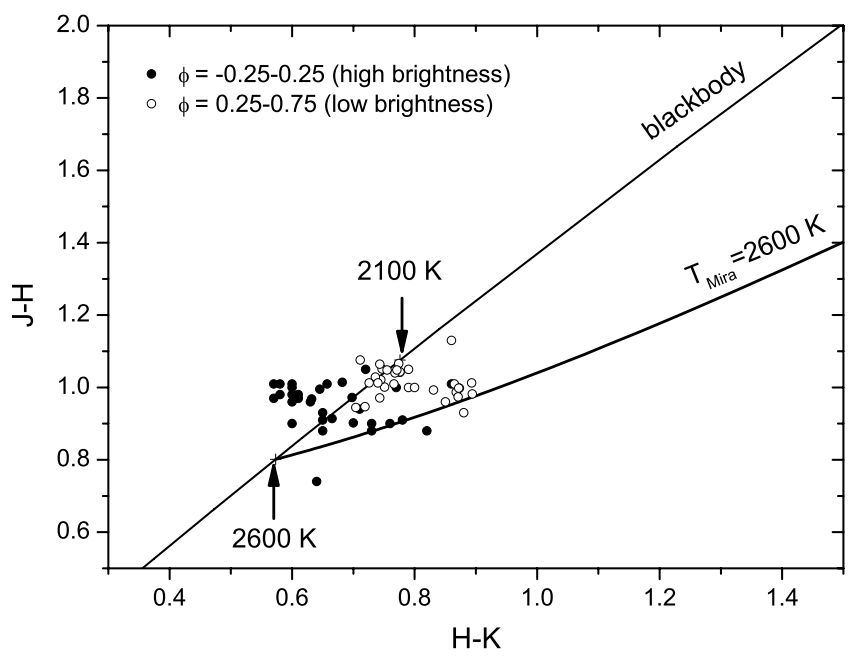

Fig. 4. Two-colour $J-H / H-K$ diagram with blackbody model used to determine the extreme temperature values of the Mira component in the epoch of minimum obscuration. The curve that intersects the blackbody line represents the typical dust shell model $\left(T_{\text {dust }}=1200 \mathrm{~K}\right)$ corresponding to the maximum temperature of the Mira component of $2600 \mathrm{~K}$. The corresponding minimum temperature is given by the uppermost observed colour indices on the blackbody curve. Observations during the half period of high brightness (phase $\phi=-0.25-0.25$ ) are given with full circles, while observations during the half period of low brightness (phase $\phi=0.25-0.75$ ) are marked by empty circles.

Table 7. Minimum, maximum, and average temperatures of the Mira component in RR Tel.

\begin{tabular}{ccc}
\hline \hline $\begin{array}{c}T_{\text {Mira }} \min \\
(\mathrm{K})\end{array}$ & $\begin{array}{c}T_{\text {Mira }} \text { avg } \\
(\mathrm{K})\end{array}$ & $\begin{array}{c}T_{\text {Mira }} \max \\
(\mathrm{K})\end{array}$ \\
\hline $2100 \pm 60$ & $2350 \pm 70$ & $2600 \pm 80$ \\
\hline
\end{tabular}

by reducing the number of free parameters in the calculation of the radiation transfer through the dust. While doing so, dependence of the Mira temperature on the pulsation phase must be taken into account because the temperature itself determines the shape of the spectra of the input radiation. The JHK SAAO magnitudes in the epoch without obscuration were used to determine the temperature of the Mira component. Because the nearinfrared part of the spectra up to $K$ band is expected to be dominated by stellar radiation of this component in the epoch when dust obscuration is negligible, the shape of the near-infrared spectra determines the temperature. To achieve this, having in mind that the $J-H$ and $H-K$ colour indices trace the relative flux in these bands, the blackbody model of stellar atmosphere was taken to fit these $J H K$ colours.

Extreme temperature values (Table 7) were determined from the corresponding uppermost and lowermost observed blackbody colours in Fig. 4. The minimum temperature can be determined directly from the uppermost observed colour indices on the blackbody curve. Because the observations corresponding to the maximum temperature are obscured and do not lie on the blackbody curve, the maximum temperature was derived from the intersection with the corresponding DUSTY model curve. This correction raises the maximum temperature for about $100 \mathrm{~K}$ relative to the temperature obtained directly from the lowermost colour indices on the blackbody line. The average temperature of $2350 \mathrm{~K}$ is slightly below the currently adopted value of $2500 \mathrm{~K}$ for this stellar type. To establish a link between the extreme values of temperature and brightness, we divided the data set into 
two groups according to the pulsation phase of each observed colour index. The first data group consists of colour indices belonging to the pulsation phases during the half period of high brightness, around the maximum magnitude. The second group has colour indices belonging to the phases during the other half period of low brightness, around the minimum magnitude. As can be seen from Fig. 4, the maximum brightness corresponds to the maximum temperature, and vice versa.

\subsection{Determination of circumstellar dust properties and dust geometry from the spectral energy distributions}

Infrared spectra of RR Tel from 1 to $13 \mu$ m were modelled with the DUSTY numerical code. We modelled only the dust regions close to the Mira component that are expected to have smallest deviation from the spherical symmetry and are least influenced by the hot companion. These dust regions have the highest densities and temperatures, near the dust sublimation temperature, and are responsible for most of the infrared emission at wavelengths of up to $8 \mu \mathrm{m}$. The silicate feature is also included in the model by extending our analysis to wavelengths of up to $13 \mu \mathrm{m}$. The regions responsible for infrared emission above $13 \mu \mathrm{m}$ are farther away from the Mira component and thus closer to the hot component, therefore they are expected to be more influenced by the hot companion, to have a more complex dust and temperature distribution, and consequently they cannot be adequately fitted by DUSTY. We used the input radiation spectra approximated by blackbody at the previously determined stellar temperature of the Mira component (Sect. 3.2) and a shell outer radius of 20 inner radii. The quasi-spherical dust shell model is expected to hold well up to $13 \mu \mathrm{m}$. Most of the dust responsible for thermal emission at wavelengths below the short wavelength side of the silicate feature $(\sim 8 \mu \mathrm{m})$ is found in the regions up to six shell inner radii from the Mira component. Note that the thermal continuum emission underlying the silicate feature at wavelengths in the range $8-13 \mu \mathrm{m}$ is weak compared with the strength of this feature. Consequently, an additional extension of the dust shell outer radius does not change the spectra significantly, because the dust present in the outer part of the shell has a low temperature and density, contributing only above $15 \mu \mathrm{m}$.

The results are given in Table 8 and Fig. 5. The dust temperature of $1200-1300 \mathrm{~K}$ in the shell inner boundary agrees, as expected, with the typical silicate dust sublimation temperature. The dust shell has a considerable optical depth, which can explain the strong obscuration in the near-infrared.

The model spectra are dominated by dust scattering up to $4 \mu \mathrm{m}$ when dust emission becomes considerable, while emission gives almost the whole contribution to the total flux above $8 \mu \mathrm{m}$. Because the amount of the scattered flux is defined by the scattering cross section, which strongly depends on the physical size of dust particles, modelling of SED in the region between 1 and $6 \mu \mathrm{m}$ will give an insight into the dust grain sizes. A similar discussion applies to the influence of the dust sublimation temperature, but on somewhat longer wavelengths, where emission becomes dominant.

Although we modelled SEDs with radial dust densities declining as $1 / y^{2}(p=2), 1 / y^{1.5}(p=1.5)$ and $1 / y(p=1)$, the best fit was achieved for a $1 / y^{2}$ radial density profile corrected for radiatively driven stellar winds (Eq. (3)). Clearly, a flatter density profile such as $1 / y$ cannot reproduce these SEDs, especially in 4-10 $\mu \mathrm{m}$ where the spectrum becomes flat and the silicate feature pronounced as more cold dust is found farther away from the Mira component. The observed SEDs prefer a steeper density distribution, which means that most of the dust is found in
Table 8. Dust shell properties determined from modelling of the reconstructed SEDs (Fig. 5) with the DUSTY numerical code.

\begin{tabular}{lccc}
\hline \hline ISO/SWS number & 54601206 & 73402079 & Average \\
Date & $1997 / 05 / 15$ & $1997 / 11 / 19$ & \\
$\mathrm{JD}$ & 2450584 & 2450772 & \\
\hline$\phi^{a}$ & $0.56(\min )$. & $0.04(\max )$. & \\
$T_{\text {Mira }}(\mathrm{K})^{b}$ & 2100 & 2600 & 2350 \\
$T_{\text {dust }}(\mathrm{K})$ & $1200_{-100}^{+100}$ & $1350_{-50}^{+150}$ & $1270_{-50}^{+80}$ \\
$a_{\max }(\mu \mathrm{m})$ & $4.0_{-0.8}^{+3.0}$ & $4.7_{-1.0}^{+2.8}$ & $4.4_{-0.6}^{+2.0}$ \\
$\tau_{\mathrm{V}}$ & $5.4_{-0.6}^{+0.2}$ & $4.8_{-0.4}^{+0.6}$ & $5.2_{-0.3}^{+0.2}$ \\
$A_{K}$ & $0.59_{-0.07}^{+0.02}$ & $0.53_{-0.04}^{+0.07}$ & $0.57_{-0.04}^{+0.02}$ \\
$r_{1} / r_{\mathrm{c}}$ & $4.1_{-0.7}^{+0.7}$ & $3.1_{-0.6}^{+0.3}$ & $3.7_{-0.4}^{+0.3}$ \\
$\dot{M}\left(10^{-6} M_{\odot} / \mathrm{yr}\right)$ & $6.3_{-0.2}^{+0.9}$ & $8.4_{-0.4}^{+1.2}$ & $7.0_{-0.2}^{+0.7}$ \\
$v_{\mathrm{e}}\left(\mathrm{km} \mathrm{s}^{-1}\right)$ & $21_{-3}^{+1}$ & $28_{-3}^{+3}$ & $25_{-2}^{+1}$ \\
$F_{\text {bol }}\left(10^{-12} \mathrm{~W} / \mathrm{m}^{2}\right)$ & 21.8 & 40.9 & 31.35 \\
$L\left(L_{\odot}\right)$ & 5100 & 9600 & $7350^{c}$ \\
\hline
\end{tabular}

Notes. JD - Julian day of the observation; $\phi$ - phase of the pulsation cycle ( $\phi=0$ for maximum brightness, $\phi=0.5$ for minimum brightness); $T_{\text {Mira }}$ - temperature of the Mira component; $T_{\text {dust }}$ - dust temperature on the inner boundary of the dust shell (dust sublimation temperature); $a_{\max }-$ maximum dust grain diameter; $\tau_{\mathrm{V}}$ - optical depth; $A_{K}-$ extinction at $K ; r_{1} / r_{\mathrm{c}}$ - radius of the shell inner boundary (dust sublimation radius) $r_{1}$ expressed in units of the stellar radius $r_{\mathrm{c}} ; \dot{M}$ - mass loss rate assuming radiatively driven winds; $v_{\mathrm{e}}$ - terminal escape velocity of the stellar wind; $F_{\text {bol }}$ - bolometric flux; $L$ - luminosity. ${ }^{(a)}$ Phases $\phi=0.56$ and $\phi=0.04$ are considered to be close enough to the values of 0.5 and 0.0 to be declared as phases of the minimum and maximum brightness. ${ }^{(b)}$ Minimum, maximum, and average stellar temperatures of the Mira component as determined in Sect. 3.2. ${ }^{(c)}$ Average luminosity of the Mira component is not derived from our SED modelling but is determined from the period-luminosity relation of Whitelock et al. (1994).

the fairly compact region close to the star, having a temperature near the sublimation temperature. Figure 6 shows a comparison between the radial dust density declining as $1 / y^{2}$ and radial density corrected for radiatively driven winds. Evidently the density declines more rapidly in the case of radiatively driven winds, which means that even more dust is found in the hottest region around the star compared to the uncorrected $1 / y^{2}$ density profile. The stellar radiation of the Mira component accelerates the dust particles, which in turn accelerate the surrounding gas due to the drag force. Hydrodynamical solutions predict low gas and dust velocities in the shell inner regions, which explains their enhanced density compared to the constant-velocity wind model. The dust density enhancement around the shell inner boundary (sublimation radius) effectively produces a compact dust shell 3-4 sublimation radii thick. Note that $85 \%$ of the total dust is found inside the 3.5 sublimation radii from the Mira star for radiatively driven winds, while for the $1 / y^{2}$ radial density profile, the same amount of dust is found inside a much larger part of the dust shell extending to more than 5 sublimation radii.

Although a substantial departure from spherical or axial symmetry might be expected because of the presence of the hot companion with a strong UV flux, our models reproduce SED spectra very well up to $13 \mu \mathrm{m}$. Even the silicate feature at $10 \mu \mathrm{m}$ is reproduced quite well, implying that most of the dust responsible for the infrared emission up to $13 \mu \mathrm{m}$ is in fact constrained in an almost spherical dust shell with small influence from the hot component. Accordingly, it is likely that some mechanism of strong UV shielding must exist, probably somewhere in the nebula between the components. 

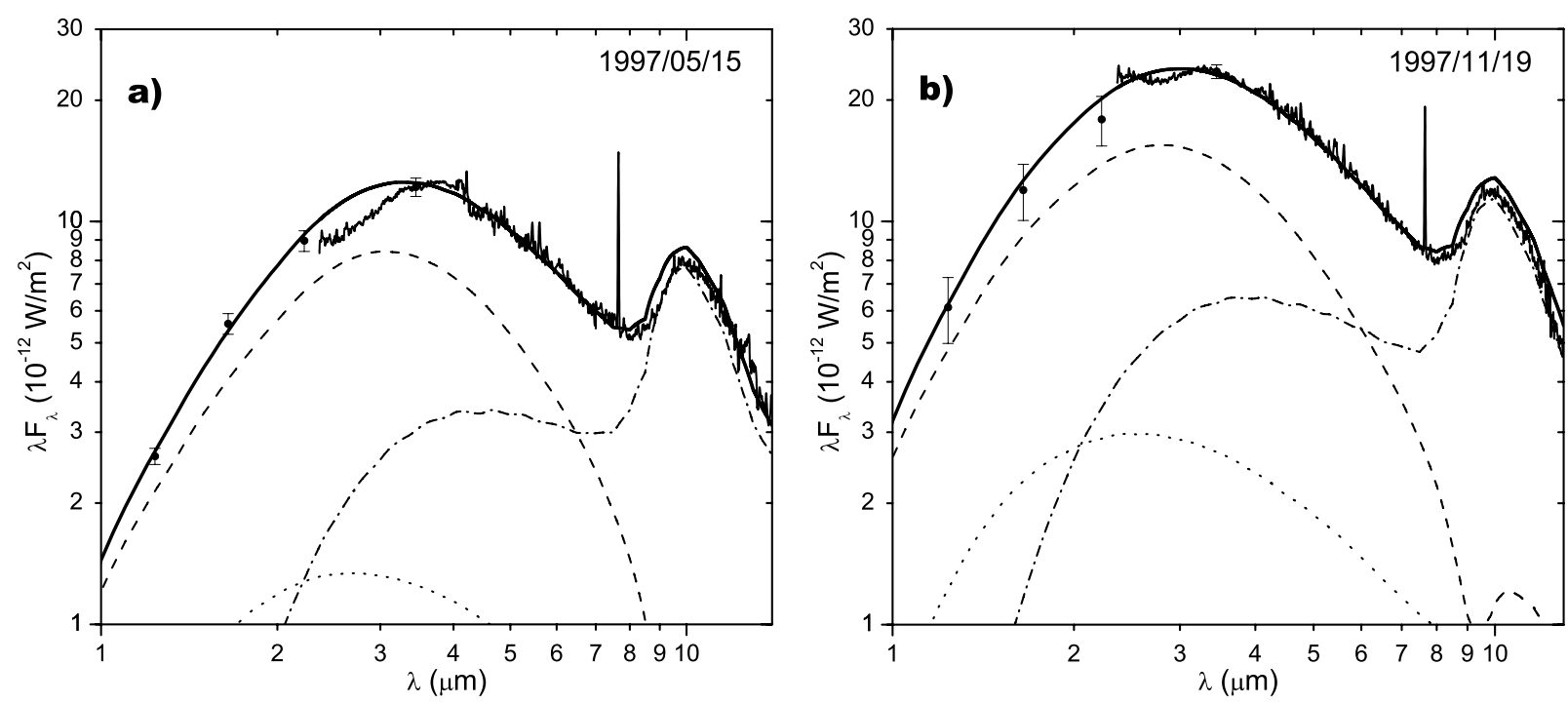

Fig. 5. Reconstructed spectral energy distributions of RR Tel in two epochs: a) 1997/05/15 and b) 1997/11/19, differing in time by a half of the Mira pulsation period. The best-fitted DUSTY model for dust density distribution enhanced by radiatively driven winds is given by the full thick line. Observations in the near-IR are marked by four circles with their corresponding error bars for $J, H, K$ and $L$ wavelengths, while the ISO/SWS observations are shown by the thin full line and cover an interval from 2.3 to $13 \mu \mathrm{m}$. The three contributions to the model are: attenuated radiation of the Mira component (dotted line), dust scattering (dashed line), and dust emission (dot-dashed line).

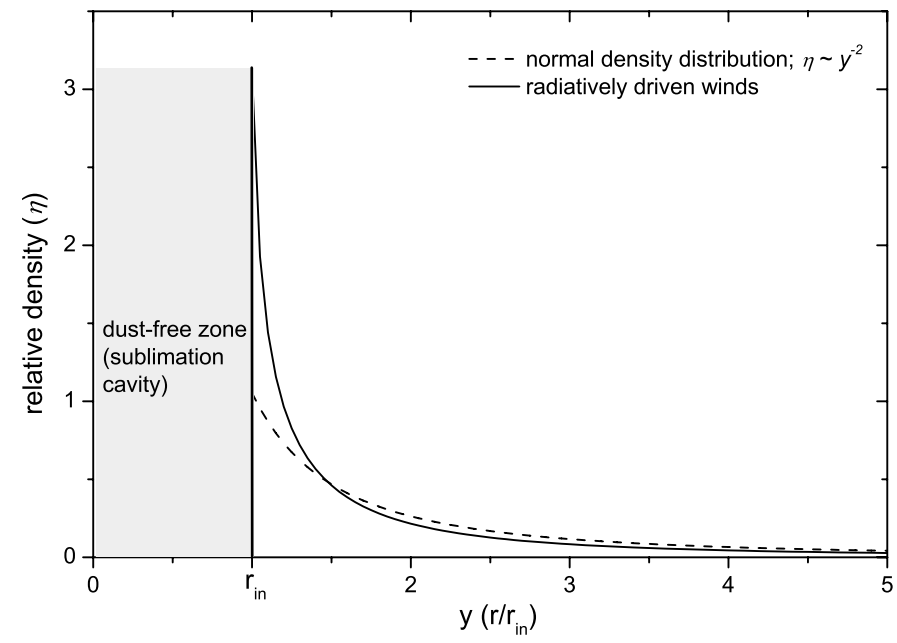

Fig. 6. Radial dust density distribution profiles for a standard density distribution declining as $1 / y^{2}$ and for density distribution enhanced by radiatively driven winds. The amount of dust is the same in both density profiles. The radial distance $r$ is scaled to the radius of the shell inner boundary (sublimation radius) $r_{\text {in }}$, giving the scaled radial distance $y$.

On the other hand, the compact dust region around the sublimation radius is formed by enhanced dust density with a temperature near its sublimation value. In such a detached system, the influence of the hot companion on this innermost and warmest part of the dust shell is expected to be low and the spectra can be satisfactorily explained by a single dust shell model.

A fairly high maximum grain size diameter of $4 \mu \mathrm{m}$ (Table 8) is needed to successfully reproduce the observed spectra, because only large grains can scatter the light effectively, and Fig. 5 shows that scattering is indispensable for RR Tel. A departure from the standard dust grain size distribution with typical grain sizes up to $0.25 \mu \mathrm{m}$ implies that the contribution of larger grains is quite significant and changes the spectral shape considerably. An increase in the relative number of larger grains can be explained by a substantial mass loss driven by a strong stellar wind. In conditions found in RR Tel, the enhanced density in the dust sublimation region is so high that it can induce the grain growth, and consequently, larger grains can be formed. An increase in maximum grain size effectively moves the grain size distribution towards larger grains, although their relative number remains rather low compared with submicron grains. On the other hand, the total mass of the larger grains becomes considerable compared with the total mass of the smaller dust particles.

The bolometric flux was obtained by integrating the bestfitted model spectra. It can be shown that the bolometric flux calculated up to $13 \mu \mathrm{m}$ consists of at least $95 \%$ of the total bolometric flux of the Mira component. Including the flux at wavelengths above $13 \mu \mathrm{m}$ does not alter the total bolometric flux by more than $5 \%$. Namely, this flux originates from the dust regions at lower temperatures and densities, farther away from the Mira component, which are expected to be more influenced by the hot companion. Evidently, an increase in total flux is accompanied by an increase in the brightness in the near-IR magnitudes, implying a higher temperature of the Mira component (Fig. 4). Thus, as expected, the total flux and luminosity increase with the Mira temperature during the pulsation cycle.

The average luminosity of the Mira component shown in Table 8 is determined from the period-luminosity (PL) relation for O-rich Miras of Whitelock et al. (1994):

$M_{\mathrm{bol}}=-3.00 \cdot \log P+2.80$,

where $M_{\mathrm{bol}}$ stands for bolometric magnitude and $P$ is the Mira pulsation period. The luminosity of the Mira component is determined by an uncertainty of $30 \%$, because the PL relation depends significantly on the sample stars and different authors give different relations. The more recent PL relation of Guandalini \& Busso (2008)

$M_{\mathrm{bol}}=-1.273 \cdot \ln P+2.348$

yields a luminosity of $9450 L_{\odot}$ compared to $7350 L_{\odot}$ from the PL relation of Whitelock et al. (1994), resulting in a difference between the two estimates of about $25 \%$. Minimum and maximum luminosities were determined from the total bolometric 
flux in the infrared using the distance to the RR Tel of $2.7 \mathrm{kpc}$ (Sect. 3.4).

The dust temperatures on the inner boundary of the dust shell (dust sublimation temperature) derived from the two SEDs at different pulsation phases (Table 8) show significant differences that cannot be attributed to statistical errors. Obviously the dust sublimation temperature increases with bolometric flux and with stellar temperature of the Mira component. The near-IR SED requires hotter dust with possibly somewhat larger grains at the maximum brightness. The solution of the problem found by DUSTY leads to an increased mass loss rate at maximum brightness, so that the physical conditions for dust formation are met closer to the stellar surface, at a smaller dust sublimation radius. This would result in dust formation at higher temperatures and, since the density is higher, in the formation of larger grains. Indeed, the results show a possible increase of the grain size diameter at the maximum brightness (Table 8), but this should be taken with caution because the grain size differences fall inside the declared errors.

Although the DUSTY models reproduce the observed spectra quite well, there are certain deviations, the largest one appearing in the shape of the near-IR dip between 2.5 and $3 \mu \mathrm{m}$, which is more pronounced during the pulsation minima. The origin of this feature can be related to different phenomena, but the most probable explanation is that it is related to a broad molecular absorption band. Because $\mathrm{CO}$ and $\mathrm{H}_{2} \mathrm{O}$ molecules can be easily formed in the cold stellar atmospheres, the absorption feature is probably caused by the $\mathrm{CO}$ transitions around $2.2 \mu \mathrm{m}$ (Wallace \& Hinkle 1996) and $\mathrm{H}_{2} \mathrm{O}$ absorption between 2.5 and $3.5 \mu \mathrm{m}$ (Yamamura et al. 1999). The fact that this near-IR absorption is more pronounced during pulsation minimum when the stellar temperature is lower speaks in favour of this explanation. On the other hand, the described feature might have its origin in the deviation from spherical symmetry caused by the binary nature of the system.

Although the separation between the components is not known, it is believed that they are at least $30 \mathrm{AU}$ apart. Lee \& Park (1999) have proposed the formation of an accretion disk around the hot component for which the separation between the components should be at least $20 \mathrm{AU}$ according to the numerical computations by Mastrodemos \& Morris (1998). Their models, which take account of the dusty wind accretion in a detached binary, show a symmetric velocity field and symmetric dust distribution if there is a considerable separation. Recent hydrodynamical calculations (Mohamed \& Podsiadlowski 2010, 2011) have proposed a new mechanism for mass transfer in symbiotic binaries that is based on the stellar wind filling its Roche lobe. Although their calculations prefer a flattened dust distribution in the plane of orbital motion, in widely detached systems their dust density distribution gives evidence of spherical symmetry. Still, the innermost and warmest part of the circumstellar dust shell close to the Mira component retains a quasi-spherical shape. On the other hand, Vinkovic et al. (2003) have shown that a flattened dust halo is equivalent to a spherical dust shell, giving rise to the same spectra. Therefore, even in the case of smaller component separation and of lower wind velocity where hydrodynamical calculations prefer flattened dust distribution in orbital plane, our analysis still holds. Note that the precise dust geometry is not particularly important as long as the dust temperature is dominated by stellar heating and has spherically symmetric distribution (Vinkovic et al. 2003).

Up to now, other authors have also tried to determine the dust properties in RR Tel considering only the dust shell approximated by a blackbody, with no attempts to use more realistic
Table 9. Luminosities and temperatures of the hot and cool components of RR Tel and HM Sge.

\begin{tabular}{lcc}
\hline \hline Parameter & RR Tel & HM Sge \\
\hline$L_{\text {cool }}\left(L_{\odot}\right)$ & $7350^{1}-9450^{2}$ & $5000^{3}-10600^{4}$ \\
$T_{\text {hot }}(\mathrm{K})$ & $140000^{5,6}$ & $100000-200000^{3,4,6}$ \\
$L_{\text {hot }}\left(L_{\odot}\right)$ & $3500^{5}-9000^{6}$ & $9000^{3,4}$ \\
\hline
\end{tabular}

Notes. ${ }^{(1)}$ PL relation of Whitelock et al. (1994). (2) PL relation of Guandalini \& Busso (2008). (3) Schild et al. (2001). (4) Bogdanov \& Tarranova (2001). ${ }^{(5)}$ Nussbaumer \& Dumm (1997), Jordan et al. (1994). (6) Muerset et al. (1991).

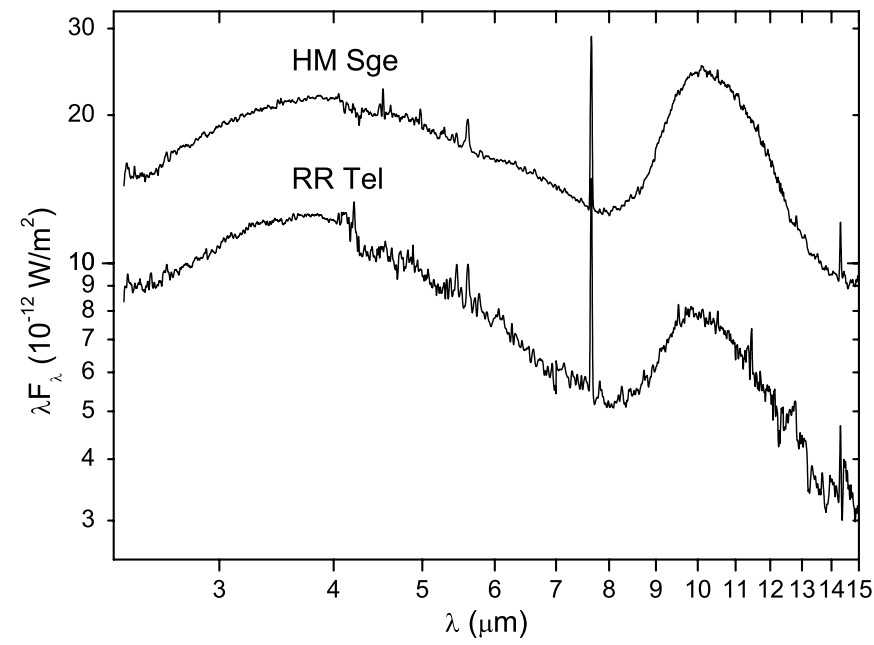

Fig. 7. Spectral energy distribution of RR Tel and HM Sge symbiotic novae in near- and mid-infrared. The similar spectral features and behaviour are evident.

dust models and to obtain its physical properties. Even Angeloni et al. (2010), who tried to model the whole spectral range, used a blackbody dust approximation. Interferometric observations of HM Sge, a symbiotic Mira similar to RR Tel, have shown a very compact and centrally symmetric dust shell up to $13 \mu \mathrm{m}$ (Sacuto et al. 2007; Sacuto \& Chesneau 2009).

To exactly determine the size and shape of the dust geometry, and to deduce the amount of influence of the hot component on the dust generated around the Mira component, interferometric observations are essential. Unfortunately, owing to the large distance to the system, no attempts have been made to observe RR Tel using infrared interferometry. Still, we can compare RR Tel with another similar symbiotic nova, HM Sge, which was observed by interferometry (Sacuto et al. 2007; Sacuto \& Chesneau 2009). Both symbiotic stars are slow novae and are widely detached systems with a separation of at least 20 AU (Lee \& Park 1999; Sacuto et al. 2007).

The luminosities and temperatures of the components in RR Tel and HM Sge are given in Table 9. Regarding the luminosity of the Mira component in HM Sge relative to RR Tel, the stellar wind of this component in HM Sge is expected to be similar to or even weaker than the corresponding wind in RR Tel. Considering the luminosities of the hot components, the influence of the hot component on the dust in HM Sge is likely to be the same or stronger relative to RR Tel. Spectral energy distributions of RR Tel and HM Sge shown in Fig. 7 are very similar and show the same spectral features that only differ in their relative intensities and slopes, which can be explained by different physical conditions in the dust shells. 
Table 10. Mass loss and distance estimate for RR Tel.

\begin{tabular}{ccc|ccccc}
\hline \hline \multicolumn{3}{c|}{$\begin{array}{c}\text { Distance } \\
(\mathrm{kpc})\end{array}$} & \multicolumn{5}{c}{$\begin{array}{c}\text { Mass loss } \\
\left(10^{-6} M_{\odot} / \mathrm{yr}\right)\end{array}$} \\
\hline This work & $\begin{array}{c}\text { Kotnik-Karuza } \\
(2006)\end{array}$ & $\begin{array}{c}\text { Whitelock } \\
(1988)\end{array}$ & This work & $\begin{array}{c}\text { Kotnik-Karuza } \\
(2002)\end{array}$ & $\begin{array}{c}\text { Whitelock } \\
(1994)\end{array}$ & $\begin{array}{c}\text { Seaquist } \\
(1993)\end{array}$ & $\begin{array}{c}\text { Kenyon } \\
(1988)\end{array}$ \\
\hline 2.7 & 2.5 & 2.6 & $7.0_{-0.2}^{+0.7}$ & $\gtrsim 3.5$ & 2.5 & 5.3 & 8 \\
\hline
\end{tabular}

Notes. Mass loss estimate for RR Tel in this work is given with an uncertainty of up to $30 \%$ introduced by the numerical code itself.

Schild et al. (2001) and Bogdanov \& Taranova (2001) used the DUSTY code to model the SEDs of HM Sge with single and double dust shells and came to opposite conclusions regarding the shell model. However, we favour the circumstellar dust model of Sacuto et al. (2007), who basically used the models of Schild et al. (2001) and Bogdanov \& Taranova (2001) to analyse both infrared SEDs and interferometric observations, clearly favouring the single dust shell model. According to them, interferometry suggests a compact single dust shell centred around the Mira component. Single shell images from interferometry up to $13 \mu \mathrm{m}$ show a surprisingly low amount of asymmetry, suggesting a significantly low influence of the hot component. Consequently, they claim that some mechanism of strong shielding from the UV flux of the hot component must exist in the ionized nebula. The shielding is probably caused by the slow and dense wind of the Mira component, which is strong enough to suppress the UV flux and to prevent the dust shell distortion as in case of the symbiotic nebula PN M2-9 (Livio \& Soker 2001).

Because HM Sge and RR Tel are similar in many features, we can expect a similar dust geometry and dust properties, which imply compact, almost spherical inner dust regions and strong UV shielding.

The complex modelling of the normal single Mira RR Aql (Karovicova et al. 2011), including both the stellar atmosphere and circumstellar dust, yielded the similar silicate composition and the dust shell inner radius of $4.1 \pm 0.7$, which is close to our value of 3.7 for RR Tel. The two values of the shell inner radii are scaled to the stellar radius.

\subsection{Mass loss and distance estimate}

We calculated the mass loss rate with the DUSTY code. The code gives the mass loss value for a luminosity of $10000 L_{\odot}$, which has to be scaled as $\dot{M} \propto L^{3 / 4}$ for the exact value of luminosity. The mass loss rate averaged over two extreme phases inside one pulsation cycle (Table 8 ) has been obtained using the luminosity value of $7350 L_{\odot}$ from the PL relation of Whitelock et al. (1994). The error of $0.7 \times 10^{-6} M_{\odot} / y r$ was derived by modelling the SEDs with DUSTY, but note that the DUSTY calculation of the mass loss rate introduces an error up to $30 \%$, which extends the error limits of the final mass loss value as $(7.0 \pm 2.1) \times 10^{-6} M_{\odot} / y r$. The corresponding mass loss rate obtained for the luminosity given by the PL relation of Guandalini $\&$ Busso (2008) has a value of $8.9 \times 10^{-6} M_{\odot} / y r$, which falls inside the upper limit of our result. Our results are in satisfactory agreement with the values of other authors (Table 10), especially with Kenyon et al. (1988), who used IRAS infrared observations.

We estimated the distance to RR Tel by calculating the average total flux from the best-fitted SED models of the two observed ISO/SWS spectra and using the average bolometric magnitude obtained from the PL relation of Whitelock et al. (1994). The estimated distance (Table 10) agrees well with the already published values. The error of our estimate is of the order of $0.3 \mathrm{kpc}$, mainly because of the uncertainty in determining the luminosity from the PL relation but not the bolometric flux.

The distance of $2.7 \mathrm{kpc}$ was derived from the infrared spectra up to $13 \mu \mathrm{m}$ assuming that the only source of radiation that heats the dust comes from the Mira component. Thus, the agreement of our value with the values of other authors support the idea that almost all dust heating comes from the Mira component only. If the heating from the hot companion were comparable with the heating from the Mira component, the bolometric flux would consequently be higher, and the system would appear closer to us assuming the luminosity determined from the PL relation.

\subsection{Determining the dust parameters from the time behaviour of the near-IR colours}

In addition to the SED modelling, the dust properties of RR Tel, with an accent on mass loss and optical depth, were determined from the behaviour of the near-IR $J H K L$ magnitudes observed from SAAO on the timescale of 20 years. The entire $J H K L$ colour indices were modelled with the DUSTY code, out of which $J-H$ and $H-L$ colour indices were selected to be plotted together with DUSTY models in the two-colour diagram (Fig. 8). The obtained dust properties are given in Table 11. Because the solutions for the pairs $\left(T_{\text {dust }}, a_{\text {max }}\right)$ in sense of minimum $\chi^{2}$ are degenerate, these parameters are represented by limits defined by degeneracy. The dust temperature and grain size obtained previously by SED modelling were taken as referent values in this procedure. The time behaviour of dust properties can be seen most easily as changes in colour indices, since they represent the ratio of fluxes at different wavelengths. An increased dust absorption in the near-infrared caused by change in dust density or opacity leads to higher absorption in the $J$ band relative to the $L$ band, which reddens and increases the colour indices. This means that the colour indices move towards the upper right side of the two-colour diagram with increasing obscuration (Fig. 8). The temporal changes in obscuration are clearly distinguishable in Fig. 9.

The observed colours show substantial scattering, which can be attributed to a change in intrinsic properties of both the Mira component and the circumstellar dust during the pulsation cycle and to the possible appearance of substructures in the dust shell. Namely, because there is no evidence that corrections of the $J H K L$ magnitudes for Mira pulsations would affect the colours substantially, the colour indices are not influenced significantly by the periodic variations in the near-IR brightness due to the stellar pulsations. Still, some reduction of scattering in colours is achieved when correcting for Mira pulsations. The corrected colour indices are used to plot the two-colour diagram shown in Fig. 8. Accordingly, because the scattering in colours cannot be remarkably reduced by correcting for Mira pulsations, to explain the remaining scattering, other phenomena should be searched 
Table 11. Dust shell properties determined by modelling JHKL colour indices with the DUSTY numerical code.

\begin{tabular}{lccccccccc}
\hline \hline $\begin{array}{l}\text { Colour } \\
\text { indices }\end{array}$ & \multicolumn{2}{c}{$T_{\text {dust }}(\mathrm{K})$} & \multicolumn{2}{c}{$a_{\max }(\mu \mathrm{m})$} & \multicolumn{2}{c}{$r_{1} / r_{\mathrm{c}}$} & $\tau_{\mathrm{V}}$ & $A_{K}$ & $\begin{array}{c}\dot{M} \\
\left(10^{-6} M_{\odot} / \mathrm{yr}\right)\end{array}$ \\
\hline$J-H / H-K$ & 1350 & $1200-1500$ & 1.5 & $0.7-6.0^{a}$ & 3.2 & $2.2-4.2$ & $1.3-6.5$ & $0.14-0.72$ & $1.6-6.9$ \\
$\left(\mathrm{~km} \mathrm{~s} \mathrm{~s}^{-1}\right)$
\end{tabular}

Notes. $T_{\text {dust }}$ - dust temperature on the inner boundary of the dust shell (sublimation temperature); $a_{\max }-$ maximum dust grain diameter; $r_{1} / r_{\mathrm{c}}-$ radius of the shell inner boundary (sublimation radius) $r_{1}$ expressed in units of the stellar radius $r_{\mathrm{c}} ; \tau_{\mathrm{V}}-$ optical depth; $A_{K}-$ extinction at $K ; \dot{M}-$ mass loss rate assuming radiatively driven winds; $v_{\mathrm{e}}$ - terminal escape velocity of the stellar wind. ${ }^{(a)}$ Upper value of the maximum grain size cannot be determined from the near-IR colours because the model is insensitive to an increase of the grain size above $\sim 6 \mu \mathrm{m}$. ${ }^{(b)}$ Errors for dust sublimation temperature, dust sublimation radius, and maximum grain size are only estimates.

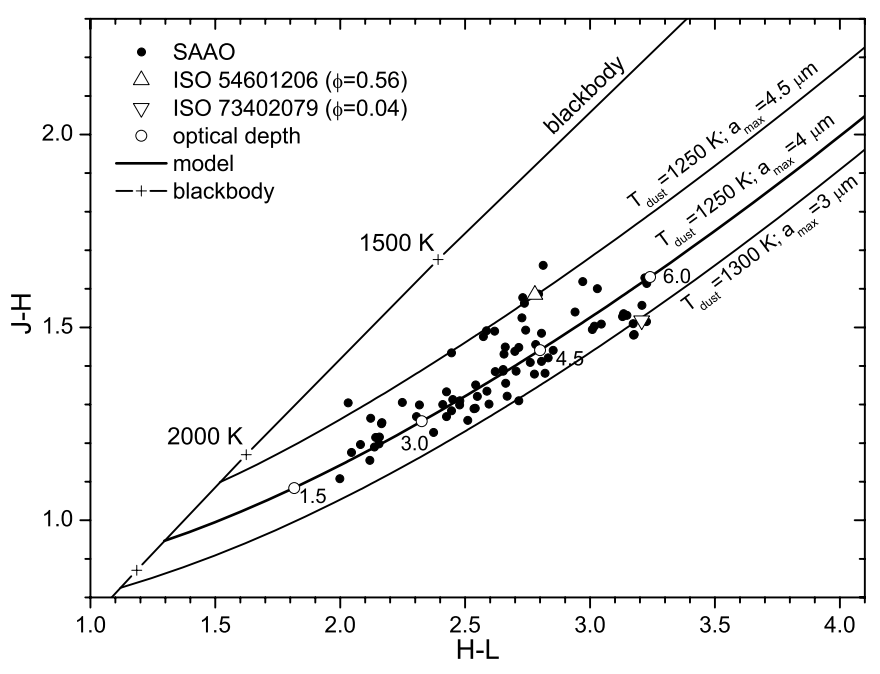

Fig. 8. Two-colour $J-H / H-L$ diagram of RR Tel with the blackbody line and fitted models. Different optical depths with numerical values are also shown on the calculated best-fitted DUSTY curve. The obtained parameters are given in Table 11.

for. Two possible candidates to explain this scattering can be suggested: a change in circumstellar dust properties such as the sublimation temperature and/or influence of a $\sim 500 \mathrm{~K}$ variation in stellar temperature on atmospheric opacity during the pulsation cycle, which introduces difficulties in the description of the input radiation by the blackbody model. Determining the dust properties only from the near-IR colours is less reliable unless other observations with wavelength coverage extended farther into infrared are available. Because only averaged flux values in $J H K L$ bands are known with no detailed spectra, modelling of circumstellar dust is a difficult task. The dominant effects on the dust in the near-IR are absorption and scattering, therefore the dust temperature and grain size diameter do not significantly affect the $J H K L$ colours. Indeed, an increase in temperature can be compensated for by an increase in grain size to give the same model in the near-IR. The only way to remove this degeneracy is to include in the analysis the observations at wavelengths beyond the $J H K L$ region with significant dust emission. Essentially, near- and mid-infrared spectra in different pulsation phases and obscuration epochs are needed. On the other hand, the optical depth of the dust and mass loss rate are much easier and more reliable to determine from the near-IR colours.

Mainly because of the explained degeneracy, we were not able to identify a substantial change of the grain size and of the dust temperature on the inner boundary of the dust shell during the investigated obscuration epoch and inside the pulsation cycle. Because Mira is a variable star that changes its luminosity, diameter, and surface temperature during the pulsation cycle, leading to changes in circumstellar temperature distribution, variations in dust temperature and radius of the shell inner boundary are likely to be expected. The dust parameters in the models that enclose the observed colour indices are consistent and cannot be considered different from the dust parameters of the models that fit the observed colours.

Among the three possible two-colour diagrams, $J-H / H-L$, $J-K / K-L$ and $H-K / K-L$, the results obtained from the $J-H$ and $H-L$ colour indices are considered to be most reliable. As can be seen from the reconstructed SEDs, the near-IR dip falls in the $K$ band, which becomes contaminated by this feature. Since molecular absorption at $K$ band cannot be modelled properly with the blackbody stellar atmosphere model, the modelling of colour indices that includes the $K$ band becomes less reliable. Indeed, dust shell properties determined from the $J-H$ and $H-L$ colour indices have values very similar to those determined by the SED modelling.

As can be seen from the colour diagram (Fig. 8), the observations follow the model line quite well as the obscuration increases or decreases. The only dust property that unambiguously does change during obscuration is the optical depth of the dust. The derived averaged dust properties, such as dust temperature on the inner boundary of the dust shell (sublimation temperature) and maximum grain diameter are consistent when different colours are modelled. The obtained optical depths also give evidence of the consistent behaviour from 1.6 to 6.3 in all colours. The results show that long-term variations in the near-IR brightness in the studied obscuration epoch can be fully understood as a result of change in optical depth of the dust. Indeed, the mass loss rate and the optical depth show a $\sim 4$-fold increase during the observed obscuration epoch (Table 11). Obscuration events with increased optical depth are expected to be caused by an increased amount of dust released into the shell from the Mira component and condensed around the sublimation radius. Indeed, hydrodynamical simulations of Mohamed \& Podsiadlowski (2011) do show this behaviour, where the dust shells are formed from the newly condensed material and are then released or ejected into the surrounding space.

\section{Conclusion}

Modelling of the spectral energy distribution in the near- and mid-infrared regions from 1 to $13 \mu \mathrm{m}$ as well as the near-infrared colours have shown that most of the warmest circumstellar dust responsible for emission in the near- and mid-IR is found in form of a compact dust shell of nearly spherical geometry in the close 


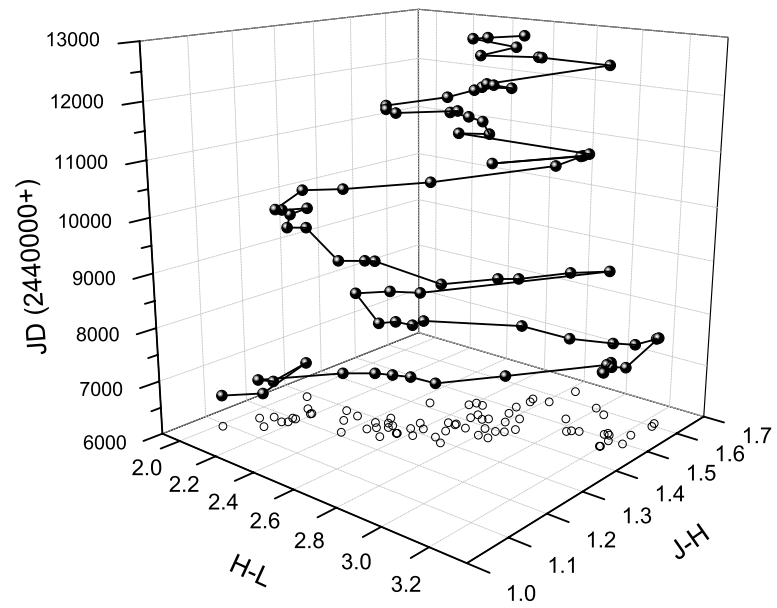

Fig. 9. Time behaviour of $J-H / H-L$ colour indices during the observed obscuration epoch from JD 2446352 to JD 2452 960. The projections on $x y$ planes are the same as the corresponding two-colour diagram in Fig. 8.

neighbourhood of the Mira component. Dust properties derived by modelling of SEDs and colour indices are consistent in the frame of the obtained errors. The typical dust sublimation temperature (i.e. the temperature on the inner boundary of the dust shell) is in the range of $1200-1300 \mathrm{~K}$, while the amount of larger grains is increased relative to the standard interstellar medium (ISM), which is dominated by smaller grain sizes. Larger grain sizes are necessary to explain the near-IR bump at $\sim 3.5 \mu \mathrm{m}$, suggesting a possible grain growth in the regime of enhanced mass loss from the Mira component. The dust obscuration events can be explained by an increase in optical depth of the dust. This effect might be caused by a new dust released from the Mira component itself followed by dust condensation around the sublimation radius. Temporal changes in the near-IR colour indices give evidence of a considerable, 4 -fold variation in the mass loss rate and optical depth during the obscuration epoch, while a significant variation of grain size and dust temperature on the shell inner boundary (sublimation temperature) was not established, neither on the long-term scale nor inside the pulsation cycle. On the other hand, modelling of the reconstructed SEDs shows a definite change in dust temperature between 1200 and $1350 \mathrm{~K}$ on the inner boundary of the dust shell at the extremes of the Mira pulsation cycle. The estimated distance of $2.7 \mathrm{kpc}$ determined from the infrared bolometric flux shows that the influence of heating from the hot companion is not significant.

Our successful model of a compact dust shell in close neighbourhood of the Mira component agrees with interferometric observations of the similar symbiotic binary HM Sge (Sacuto et al. 2007) and is supported by hydrodynamical simulations for widely separated binaries (Mohamed \& Podsiadlowski 2011). Consequently, the influence of the UV flux from the hot companion must be reduced by some sort of shielding mechanism.

To constrain the dust properties and the dust geometry more tightly, more observations are needed. Infrared interferometry has proved to be the most valuable tool for determining the geometry of the dust distribution. Unfortunately, the large distance to RR Tel is an obstacle in applying this powerful method. Although the influence of the hot component is likely to be less important, further improvement of the numerical code is needed to include the external dust heating and to check against any significant differences.
Acknowledgements. Our thanks are due to P. Whitelock and M. Gromadzki for providing us with the near-IR photometric observations of RR Tel. We also express our gratitude to anonymous referee for carefully reading the manuscript and helping us to improve and clarify the presentation.

\section{Appendix A: Reconstruction of the spectral energy distribution}

The reconstruction of the SED from the ISO/SWS spectra and $J H K L$ SAAO photometric observations of RR Tel was carried out with the following procedure. The integrated $J, H, K$ and $L$ fluxes $F_{J H K L}^{\mathrm{Vega}}$ of Vega were calculated from

$F_{J H K L}^{\mathrm{Vega}}=\frac{\int F_{\lambda}^{\mathrm{Vega}} R_{\lambda} \mathrm{d} \lambda}{\int R_{\lambda} \mathrm{d} \lambda}$,

where $F_{\lambda}^{\text {Vega }}$ is Vega flux at wavelength $\lambda$ and $R_{\lambda}$ is the transmission coefficient for the selected filter.

Integrated flux $F_{\mathrm{L}}^{\mathrm{ISO}}$ at $L$ band obtained from the ISO/SWS spectra is

$F_{\mathrm{L}}^{\mathrm{ISO}}=\frac{\int_{\mathrm{L}} F_{\lambda}^{\mathrm{ISO}} R_{\lambda} \mathrm{d} \lambda}{\int_{\mathrm{L}} R_{\lambda} \mathrm{d} \lambda}$,

where $F_{\lambda}^{\mathrm{ISO}}$ is the ISO/SWS flux at wavelength $\lambda$, while the photometric magnitude $m_{\mathrm{L}}^{\mathrm{ISO}}$ at $L$ determined from ISO/SWS spectra is

$m_{\mathrm{L}}^{\mathrm{ISO}}=-2.5 \log \frac{F_{\mathrm{L}}^{\mathrm{ISO}}}{F_{\mathrm{L}}^{\text {Vega }}}+0.026$.

Finally, the fluxes at $J, H, K$ and $L$ bands determined from the SAAO photometry are

$F_{J H K L}^{\mathrm{SAAO}}=F_{\mathrm{L}}^{\mathrm{ISO}} \frac{F_{J H K L}^{\mathrm{Vega}}}{F_{\mathrm{L}}^{\text {Vega }}} 10^{-0.4\left(m_{J H K L}-m_{\mathrm{L}}\right)}$,

where $m_{J H K L}$ are the $J, H, K$ and $L$ SAAO photometric magnitudes, respectively, and $m_{L}$ is the SAAO magnitude at $L$ band.

\section{Appendix B: The fitting procedure for the spectral energy distribution}

The DUSTY numerical code calculates normalized $f_{\lambda}$ values where $f_{\lambda}^{\text {theory }}=\lambda F_{\lambda}^{\text {theory }} / F_{\text {bol }}$ and $F_{\text {bol }}=\int F_{\lambda} \mathrm{d} \lambda$, while $F_{\lambda}^{\text {theory }}$ represents the theoretical flux from DUSTY. The theoretical fluxes must be reddened for interstellar extinction:

$f_{\lambda}^{\text {theory }}=f_{\lambda \text {,noextinction }}^{\text {theory }} 10^{-0.4 A_{\lambda}}$

where $A_{\lambda}$ is interstellar extinction at wavelength $\lambda$.

The scaling factor between the theoretical DUSTY fluxes and the observed ISO/SWS and JHKL SAAO fluxes is the bolometric flux $F_{\text {bol }}$ :

$\lambda F_{\lambda}^{\mathrm{ISO}}=F_{\text {bol }} f_{\lambda}^{\text {theory }} \quad F_{J H K L}^{\mathrm{SAAO}}=F_{\text {bol }} \frac{f_{J H K L}^{\text {theory }}}{\lambda_{J H K L}}$,

where $\lambda_{J H K L}=\int \lambda R_{\lambda} \mathrm{d} \lambda / \int R_{\lambda} \mathrm{d} \lambda$ represents the mean wavelength for each of the $J, H, K$ and $L$ bands. The integrated theoretical DUSTY fluxes $F_{J H K L}^{\text {theory }}$ at the $J, H, K$ and $L$ bands are calculated according to

$F_{J H K L}^{\text {theory }}=\frac{\int \frac{f_{\lambda}^{\text {theory }}}{\lambda} R_{\lambda} \mathrm{d} \lambda}{\int R_{\lambda} \mathrm{d} \lambda}$. 
Fitting is performed in $\log \left(f_{\lambda}\right)-\log (\lambda)$ space, so that $F_{\text {bol }}$ is given by

$$
\begin{aligned}
\log F_{\mathrm{bol}} & =\sum_{i=1}^{n} w_{i}\left[\log \lambda F_{\lambda}^{\mathrm{ISO}}-\log f_{\lambda}^{\text {theory }}\right] \\
& +\sum_{i=J, H, K, L} w_{i}\left[\log \lambda_{J H K L} F_{J H K L}^{\mathrm{SAAO}}-\log \lambda_{J H K L} F_{J H K L}^{\text {theory }}\right]
\end{aligned}
$$

where $w_{i}$ stands for statistical weight for each ISO/SWS observation and $J, H, K$, and $L \mathrm{SAAO}$ magnitude; $n$ is the number of ISO/SWS observations.

Equation (B.4) in log space leads to

$$
\log F_{\text {bol }}=\log \prod_{i=J, H, K, L}{\frac{F_{J H K L}^{\mathrm{SAAO}}}{F_{J H K L}^{\text {theory }}}}^{\frac{w_{i}}{\sum_{i=1}^{N} w_{i}}} \prod_{i=1}^{n}{\frac{\lambda_{i} F_{\lambda}^{\mathrm{ISO}}}{f_{\lambda}^{\text {theory }}}}^{\frac{w_{i}}{\sum_{i=1}^{N} w_{i}}},
$$

where the statistical weights are normalized to the total statistical weight $\sum_{i=1}^{N} w_{i}$, including the statistical weights of ISO/SWS observations and of $J, H, K$ and $L$ fluxes calculated from the near-IR photometry.

Statistical weights of ISO/SWS observations are set to 1 , while the statistical weights of the $J, H, K$ and $L$ fluxes derived from the SAAO photometry are calculated from the mean bandwidths $W_{J H K L}=\int R_{\lambda} \mathrm{d} \lambda$ for each of the $J, H, K$ and $L$ bands, divided by wavelength interval of ISO/SWS observations $\Delta \lambda$ :

$w_{J H K L}=\frac{W_{J H K L}}{\Delta \lambda}$.

The above procedure removes the distortion of the fitted results caused by the large difference in the number of observational data in different wavelength intervals.

All DUSTY theoretical fluxes are scaled to observed values by the bolometric flux $F_{\text {bol }}$. Finally, $\chi^{2}$ is determined as

$\chi^{2}=\sum_{i=1}^{n} w_{i} \log ^{2}\left(\frac{\lambda F_{\lambda}^{\mathrm{ISO}}}{F_{\text {bol }} f_{\lambda}^{\text {theory }}}\right)+\sum_{i=J, H, K, L} w_{i} \log ^{2}\left(\frac{F_{J H K L}^{\mathrm{SAAO}}}{F_{\text {bol }} F_{J H K L}^{\text {theory }}}\right)$.

\section{References}

Allen, D. A. 1980, MNRAS, 192, 521

Angeloni, R., Contini, M., Ciroi, S., \& Rafanelli, P. 2010, MNRAS, 402, 2075

Belczynski, K., Mikolajewska, J., Munari, U., Ivison, R. J., \& Friedjung, M. 2000, A\&AS, 146, 407

Bessell, M. S., \& Brett, J. M. 1988, PASP, 100, 1134

Bogdanov, M. B., \& Taranova, O. G. 2001, AZh, 78, 52

Bohlin, R. C. 2007, in The Future of Photometric, Spectrophotometric, and Polarimetric Standardization, ed. C. Sterken (San Francisco: ASP), ASP Conf. Ser., 364, 315
Bryan, G. L., \& Kwok, S. 1991, ApJ, 368, 252

Carter, B. S., 1990, MNRAS, 242, 1

Contini, M., \& Formiggini, L. 1999, ApJ, 517, 925

Elitzur, M., \& Ivezic, Z. 2001, MNRAS, 327, 403

Feast, M. W., Whitelock, P. A., Catchpole, B. S., Roberts, G. R. M., \& Carter, B. S. 1983, MNRAS, 202, 951

Glass, I. S. 1973, MNRAS, 164, 155

Gromadzki, M., Mikolajewska, J., Whitelock, P., \& Marang, F. 2009, Acta Astron., 59, 2, 169

Guandalini, R., \& Busso, M. 2008, A\&A, 488, 675

Ivezic, Z., \& Elitzur, M. 1997, MNRAS, 287, 799

Ivezic, Z., Nenkova, M., \& Elitzur, M. 1999, in User Manual for DUSTY, University of Kentucky Internal Report,

http://www .pa.uky.edu/ moshe/dusty

Jordan, S., Muerset, U., \& Werner, K. 1994, A\&A, 283, 475

Karovicova, I., Wittkowski, M., Boboltz, D. A., et al. 2011, A\&A, 532, A134

Kenyon, S. J. 1986, in The Symbiotic Stars (Cambridge: CUP), 295

Kenyon, S. J., Fernandez-Castro, T., \& Stencel, R. E. 1988, AJ, 95, 1817

Kotnik-Karuza, D., Friedjung, M., \& Selvelli, P. L. 2002, A\&A, 381, 507

Kotnik-Karuza, D., Friedjung, M., Whitelock, P. A., et al. 2006, A\&A, 452, 503

Lafler, J., \& Kinman, T. D. 1965, ApJS, 11, 216

Lee, H.-W., \& Park, M.-G. 1999, ApJ, 515, L89

Livio, M., \& Soker, N. 2001, ApJ, 552, 685

Mastrodemos, N., \& Morris, M. 1998, ApJ, 497, 303

Mathis, J. S., Rumpl, W., \& Nordsieck, K. H. 1977, ApJ, 217, 425

Mayall, M. W. 1949, Harvard Bull., 919, 15

Mikolajewska, J., Friedjung, M., Kenyon, S. J., \& Viotti, R. 1988, in The symbiotic phenomenom, Proc. IAU Colloq., 103 (Dordrecht: Kluwer), 145, 381

Mohamed, S., \& Podsiadlowski, Ph. 2010, in International Conference on Binaries, AIP Conf. Proc., 1314, 51

Mohamed, S., \& Podsiadlowski, Ph. 2011, in Asymmetric Planetary Nebula 5 conference, eds. A. A. Zijlstra, F. Lykou, I. McDonald, \& E. Lagadec (Jodrell Bank Centre for Astrophysics), 295

Muerset, U., \& Schmid, H. M. 1999, A\&AS, 137, 473

Muerset, U., Nussbaumer, H., Schmid, H. M., \& Vogel, M. 1991, A\&A, 248, 458

Nussbaumer, H., \& Dumm, T. 1997, A\&A, 323, 387

Ossenkopf, V., Henning, Th., \& Mathis, J. S. 1992, A\&A, 261, 567

Penston, M. V., Benvenuti, P., Cassatella, A., et al. 1983, MNRAS, 202, 833

Sacuto, S., \& Chesneau, O. 2009, A\&A, 493, 1043

Sacuto, S., Chesneau, O., Vannier, M., \& Cruzalebes, P. 2007, A\&A, 465, 469

Schild, H., Eyres, S. P. S., Salama, A., \& Evans, A. 2001, A\&A, 378, 146

Seaquist, E. R., Krogulec, M., \& Taylor, A. R. 1993, ApJ, 410, 260

Sloan, G. C., Kraemer, K. E., Price, S. D., \& Shipman, R. F. 2003, ApJS, 147, 379

Thackeray, A. D. 1950, MNRAS, 110, 45

Tuthill, P. G., Danchi, W. C., Hale, D. S., Monnier, J. D., \& Townes, C. H. 2000, ApJ, 534, 907

Vinkovic, D., Ivezic, Z., Miroshnichenko, A. S., \& Elitzur, M. 2003, MNRAS, 346,1151

Vinkovic, D., Ivezic, Z., Jurkic, T., \& Elitzur, M. 2006, ApJ, 636, 348

Wallace, L., \& Hinkle, K. 1996, ApJS, 107, 312

Whitelock, P. A. 1988, in The Symbiotic Phenomenon, eds. J. Mikolajewska, M. Friedjung, S. J. Kenyon, \& R. Viotti (Dordrecht: Kluwer Academic Publishers), 47

Whitelock, P. A. 2003, in Symbiotic stars probing stellar evolution, eds. R. L. M. Corradi, J. Mikolajewska, \& T. J. Mahoney (San Francisco: ASP), ASP Conf. Ser., 303, 41

Whitelock, P. A., Menzies, J., Feast, M., et al. 1994, MNRAS, 267, 711

Yamamura, I., de Jong, T., \& Cami, J. 1999, A\&A, 348, L55

Young, P. R., Berrington, K. A., \& Lobel, A. 2005, A\&A, 432, 665 